\title{
Bibliometric Review of Mental Health Research in Medical Colleges of Pakistan
}

${ }^{*}$ Rai K. Farooq, ${ }^{1}$ Zohaib Syed,${ }^{2}$ Ali Zulqernain ${ }^{3}$

$$
\text { مراجعة بيبليومترية لأبحاث الصحة النفسية في الكليات الطبية في باكستان }
$$

$$
\text { راي خالد فاروق، زوهيب سيد، علي ذو القرنين }
$$

ABSTRACT: Objectives: Mental health is a less frequently explored area of medical research as both developing and developed countries lack competent human resources and funding for this purpose. Reviewing mental health research can help medical professionals appreciate the progress of understanding and identify problems in this area. This systematic review examined the status of mental health research carried out in medical education institutions and tertiary healthcare hospitals across Pakistan over the past 70 years. Methods: PubMed ${ }^{\mathbb{B}}$ (National Library of Medicine, Bethesda, Maryland, USA), was searched for articles published between 1947 and 2017 related to mental health with an emphasis on the exclusive affiliation of the first author with a medical college in Pakistan. Results: A total of 118 articles were included in this study. The number of published research-based studies has increased steadily over the past years. However, there are indicators of a lack of quality research, such as no declaration of conflict of interest or the identification of a funding source and a general lack of publications in a journal with a high impact factor. Conclusion: The findings of this study have shown a steady improvement in the quantity and quality of mental health research conducted in Pakistani medical colleges/universities. However, a lack of funding, training and faculty induction policies may be hindering the establishment of a research culture and contributing to the slow progression of mental health research in Pakistan.

Keywords: Medical Education; Mental Health; Research; Psychiatry in Literature; Pakistan.

الملخص: الههف: الصحة النفسية هي مجال أقل سبرا في الأبحاث الطبية حيث تفتقر كل من البلدان النامية والمتقدمة إلى الموارد البثرية

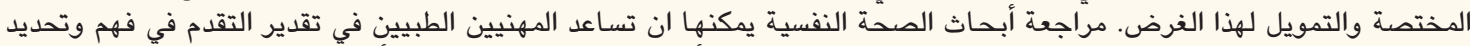

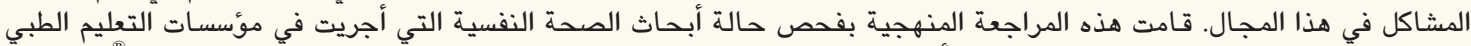

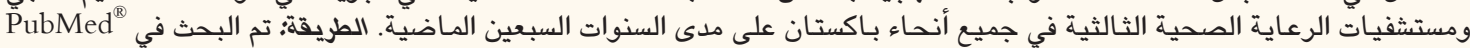

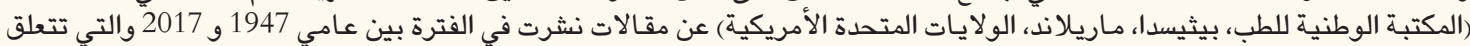

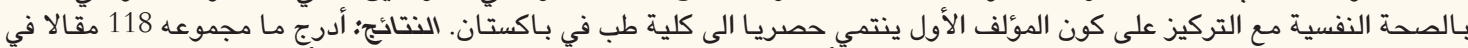

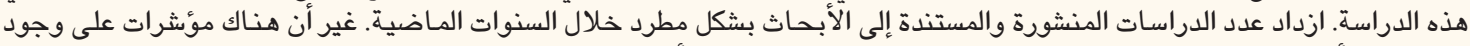

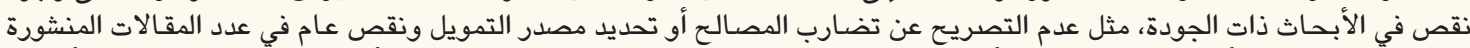

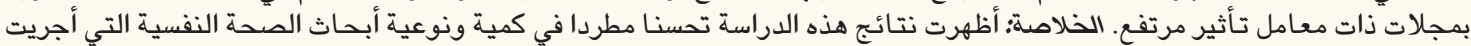

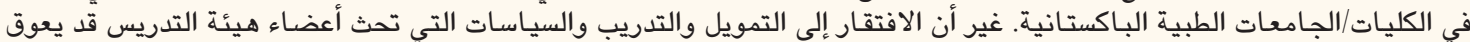
إقامة ثقافة بحثية ويساهم في التقدم البطيء لأبحاث الصاثية الصحة النفسية في بـاكستان.

$$
\text { الكلمات المفتاحية: التعليم الطبي؛ الصحة النفسية؛ ابحاث؛ الطب النفسي في الأدبيات العلمية؛ بـاكستان. }
$$

\section{Advances in KNOWLEDGE}

The findings of this analysis highlight an increase in the quantity of mental health research from Pakistan but has also found gaps in policy and practice that need to be addressed to improve the quality and depth of the research.

The results of the study show that more clinicians need to be educated about declaring conflict of interest, patient informed consent and disclosure of funding.

\section{Application to Patient Care}

This study highlights the most important area currently under examination in Pakistan, depression and other mood disorders, which are also the most prevalent psychiatric disorders in the country.

Policy changes are needed to enable the training of more physicians and scientists, which will improve Pakistan's research output and contribute to improving patient care. 
A

SUCCESSFUL HEALTHCARE SYSTEM IS CHARACterised by vision and strategy, flexibility and autonomy in decision-making, path dependency, as well as synergy and harmony with cultural preferences. ${ }^{1,2}$ Evidence-based medical research contributes significantly to the well-being of a society; thus, medical research is considered mandatory for a well-functioning healthcare system. ${ }^{3,4}$ However, the establishment of a research culture is inhibited in many countries by its low prioritisation and budget constraints. For example, Pakistan spends less than 3\% of its gross national budget on healthcare. ${ }^{5}$ In addition to restricted resource allocation, an environment that promotes healthcare research is also lacking. There is a dearth of research output from developing countries and the quality of research has been reported as low, leading to an underrepresentation in leading international medical journals. ${ }^{6}$

The impact of medical research journals originating from developing countries is also low. Only approximately $2 \%$ of journals published in developing countries are cited in the Science Citation Index (Clarivate Analytics, Philadelphia, USA). ${ }^{7}$ In addition, technological advances have further widened the healthcare research gap between developed and developing countries. ${ }^{8}$ Monitoring such trends in medical publication of scientific literature can help evaluate progress and the development of policies. ${ }^{9}$

Mental health research is one of the most neglected and undervalued areas in health policy, funding and research in developing countries, where communicable diseases are prioritised. ${ }^{10}$ In developing countries, the scarcity of mental health resources, including the capacity for conducting research, adversely contributes to this crisis. ${ }^{11}$ Middle- and low-income countries represent $90 \%$ of the world's population; however, these countries account for only $6 \%$ of the literature published in the world's six leading mental health journals. ${ }^{12}$

In 2009, Pakistan was $122^{\text {nd }}$ in the World Health Organization's ranking of healthcare systems and in the provision of healthcare services. ${ }^{13}$ This ranking is worrying for a country which ranks sixth in terms of population. The scarcity of allocated resources for mental healthcare is reflected in Pakistan's low research output. Despite a considerable number of medical education institutions in the country, research training and research output do not meet the aims of healthcare policy and planning by the Pakistan Medical Research Council. ${ }^{14}$

Therefore, this review aimed to assess the qualitative and quantitative aspects of mental health research originating from medical education institutions in Pakistan.

\section{Methods}

Pubmed $^{\mathbb{R}}$ (National Library of Medicine, Bethesda, Maryland, USA) was used to search for mental health articles published from 1947 to 2017 originating from medical colleges/universities in Pakistan. Multiple searches were performed where the search terms "Pakistan" and "mental health" were paired with "medical college", "medical university" or "health sciences". Mental health research terms such as "depression", "depressive disorder", "bipolar affective disorder", "psychosis" and "schizophrenia" were also included in order to refine the search. Only articles related to mental health where the first author was affiliated exclusively with a medical college/university (public or private) in Pakistan were included. Publications where the first author had dual or multiple affiliations were excluded as any one of the other affiliations could have been with institutions outside of Pakistan. In addition to quantifying the number of articles published per year, the known parameters of research quality (e.g. the type and design of the study, impact factor of the journal, declaration of source of funding and conflict of interest) were analysed.

\section{Results}

In total, 458 mental health articles were identified and 246 duplicates were removed from the analysis. Of the 212 remaining publications, 94 were excluded as the first author was not exclusively affiliated with a medical college/university in Pakistan. Finally, a total of 118 articles were included in this study [Figure 1].

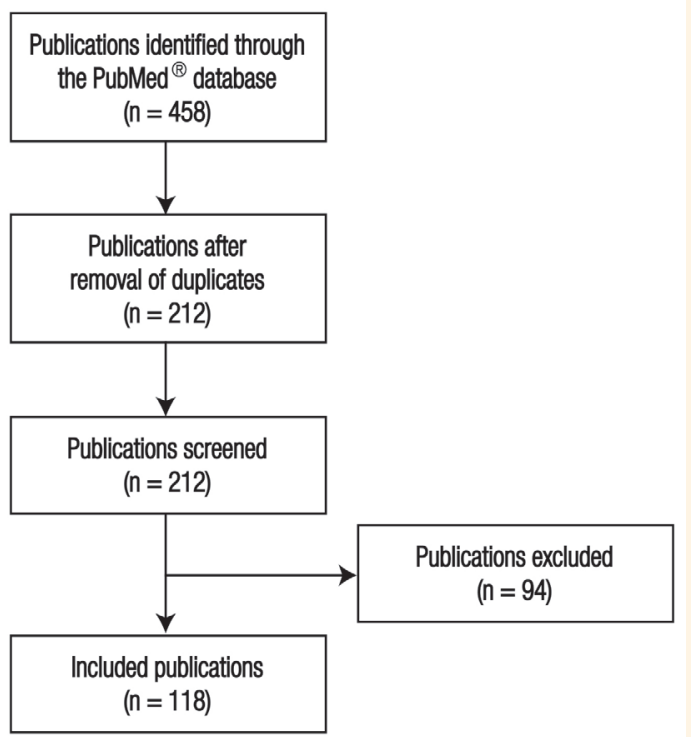

Figure 1: Diagram of the methodology showing the number of publications identified, screened and included in the current study. 
A

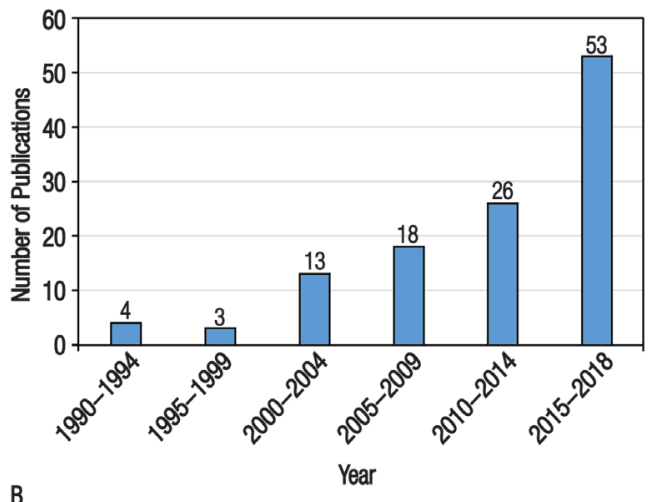

B

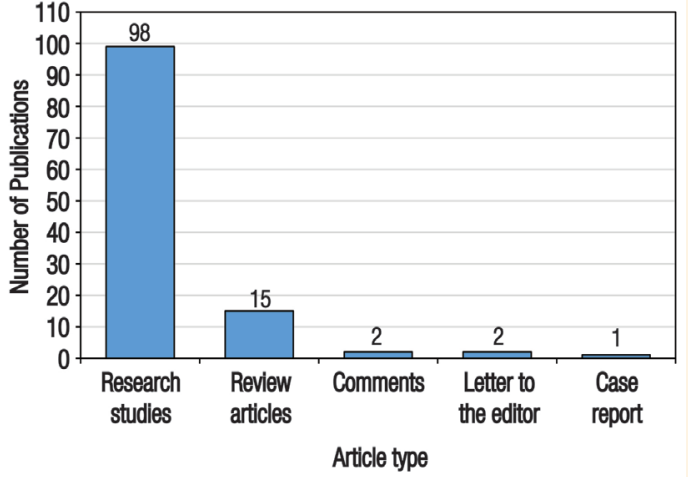

Figure 2: A: Number of publications from 1947 to 2017 about mental health from first authors affiliated with a medical institution in Pakistan. B: Distribution of the types of articles published between 1947 and $2017(\mathrm{~N}=118)$.

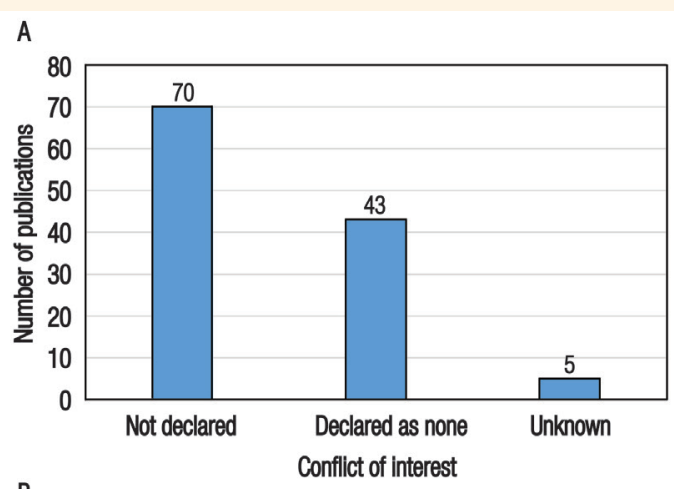

B

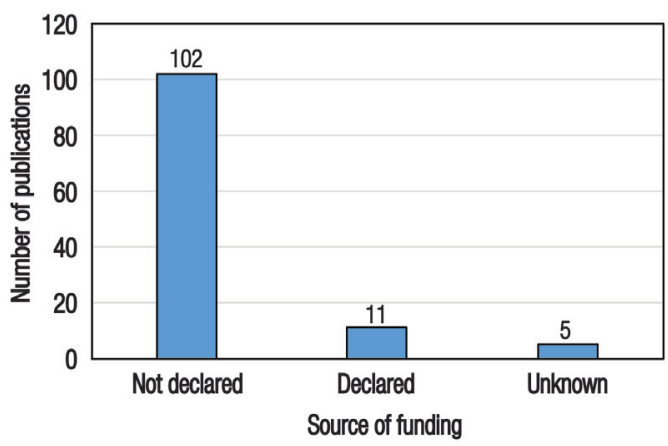

Figure 3: Distribution of the frequency of declaration of (A) any conflict of interest and (B) source of funding of articles published between 1947 and 2017 about mental health where the first author was affiliated with a medical institution in Pakistan ( $\mathrm{N}=118)$.
A

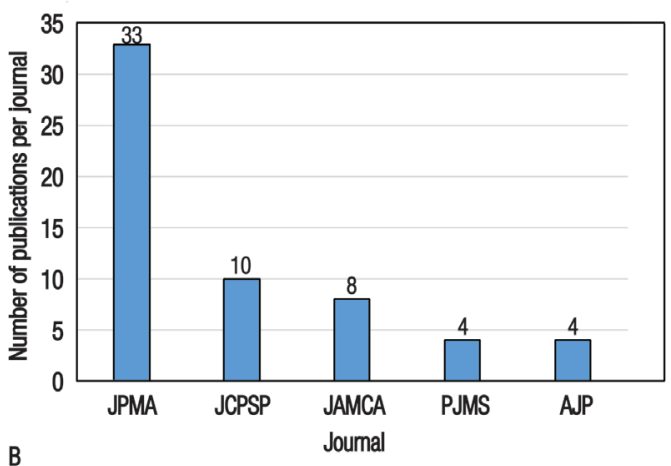

B

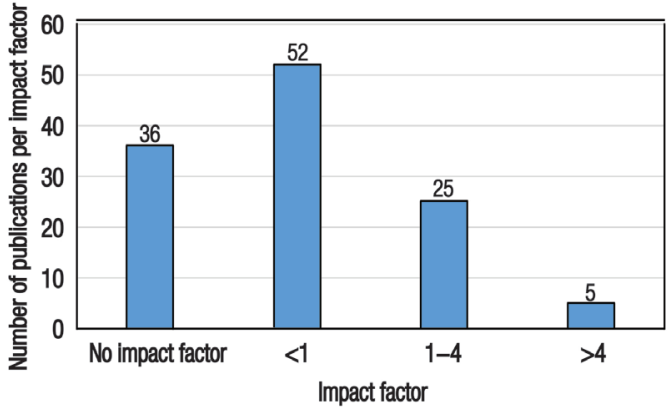

Figure 4: A: Five journals with the highest number of publications included in the current study $(\mathrm{n}=59)$. B: Frequency of publications per impact factor of the publishing journals $(\mathrm{N}=118)$.

$J P M A=$ Journal of Pakistan Medical Association; JCPSP = Journal of College of Physicians $\&$ Surgeons of Pakistan; JAMCA = Journal of Ayub Medical College Abbottabad; PJMS = Pakistan Journal of

Medical Sciences; AJP = Asian Journal of Psychiatry.

The number of mental health articles from Pakistan has risen steadily since 1993 and more than half (55.9\%) of the included articles were published after 2013 [Figure 2A]. The majority of articles (83.9\%) were research studies of which more than half (56.6\%) were studies examining the prevalence of different symptoms in different segments of the population, including medical students, hospital staff and patients admitted to hospitals with medical or surgical comorbidities not directly related to mental health including chronic illness. Review articles were the second most common type of publication (12.7\%) [Figure 2B].

Among the crucial parameters that were severely lacking in the included articles were the disclosure of financial aid and conflicts of interest. Funding sources were not listed in $86.4 \%$ of studies while conflict of interest was withheld in 59.3\% of studies; $9.3 \%$ of studies declared their source of funding and 36.4\% stated the absence of a conflict of interest [Figure 3].

Another aspect that determines quality of research is the impact factor of the publishing journal, which is an indirect measure of a journal's readership..$^{15}$ The two journals that published most of the included studies were the Journal of Pakistan Medical Association (impact factor: $0.718 ; 27.9 \%$ ) and the Journal of College 


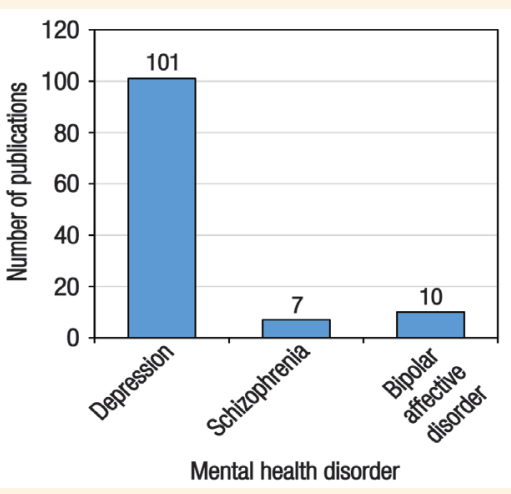

Figure 5: Frequency of publications about different areas of mental health included in the current study $(\mathrm{N}=118)$.

of Physicians and Surgeons Pakistan (impact factor: $0.372 ; 8.5 \%$ ) [Figure 4A]. In total, $69.5 \%$ of studies were published in journals with an impact factor, but a total of $44.1 \%$ were published in journals with impact factors less than one [Figure 4B].

Most studies (85.6\%) were related to depression. There was only one research article published on bipolar affective disorder in 1991, yet seven review articles were published on this subject by a single author in the span of four years (2014-2017). In addition, seven articles were found using the keyword "schizophrenia" [Figure 5]. None of these seven articles on schizophrenia declared funding sources and only one declared an absence of a conflict of competing interests.

\section{Discussion}

An evaluation of the overall status of mental health research can serve as a valuable indicator of whether a country's policy aims and goals have been met. This study highlights Pakistan's achievements and shortcomings in establishing a research culture in its medical education institutions during the previous 70 years. In addition, this study focused on affiliated medical colleges/universities and their potentially valuable contributions to the country's medical research. The current results are in accordance with previously published reviews on this subject. ${ }^{16}$

The results of this study indicate a steady improvement in the parameters of a quality research culture. However, based on these results and given Pakistan's population of almost 200 million and a minimum of 400 psychiatrists in teaching hospitals, medical colleges or private practices, an average of four publications per year is a relatively low research output.

Various factors may account for the low quantity and quality of research output in medical colleges/ universities in Pakistan; budget allocation for health and medical research is the most important issue in this regard. Of the studies that declared a source of funding, none were funded by the public sector, which may reflect the low access of Pakistani medical professionals to research funds. This may show a lack of interest by mental health practitioners, researchers and public sector funding agencies in creating an environment that supports securing grants. For this reason, medical education institutions have not been able to benefit from available research funding opportunities. No study included in the current study declared benefitting from the National Research Program for Universities provided by the Higher Education Commission of Pakistan. This loss of financial support opportunities could be due to insufficient expertise to convince grant reviewers and/or a lack of commitment by the researchers.

Certain faculty induction policies by the country's regulatory authority, the Pakistan Medical and Dental Council (PMDC) may be contributing to the lack of quality and quantity of publications. The PMDC has imposed a ban on recruiting lecturers and professors without a Bachelor of Medicine/Bachelor of Surgery to teach in medical colleges and teaching hospitals across Pakistan. ${ }^{17}$ This policy deprives researchers without basic medical degrees the opportunity to work in medical colleges.

Developing countries, including Pakistan, are plagued by corruption and unfair policies which are counterproductive to the healthcare sector. ${ }^{18}$ The lack of commitment to establishing a supportive research environment can also be seen in the absence of well-equipped molecular biology and biotechnology departments in most medical colleges and universities across Pakistan. ${ }^{19}$ In addition, public sector colleges and universities lack funding mechanisms for research. The website of the oldest and most prestigious seat of medical learning in Pakistan, King Edward Medical University, for example, has no mention of any funding mechanism to support research projects in the university at undergraduate or postgraduate level. ${ }^{19}$

The most popular post-graduate clinical training programmes are those offered by the College of Physicians and Surgeons Pakistan (CPSP); this institution offers post-graduate degrees in clinical specialties and subspecialties. ${ }^{20}$ The CPSP requires its clinical fellows to acquire research skills and submit a research dissertation in order to be able to take the fellowship's exit examination..$^{21}$ Although this college offers fellowships in basic medical sciences, it still has no system for providing research funding. Clinicians who are primarily focused on clinical excellence cannot dedicate themselves to research training and conducting projects, partly due to the absence of funding. Without funding, 
there is a risk of compromising the quality of a research dissertation. ${ }^{22}$ In addition, there is a dearth of research capacity due to a lack of infrastructure, absence of physician-scientists, a shortage of training in research and a lack of competent supervision. ${ }^{23}$ Reforming this system will require deeper understanding and commitment to necessary changes. ${ }^{24}$

The significance of physician-scientists has been acknowledged in both general and mental health research. ${ }^{25}$ Building and retaining a cohort of involved individuals in this area of expertise can be achieved by broadening the focus of healthcare and initiating research programmes. ${ }^{26}$ Research-oriented policies, better research funding facilities and an encouraging and enabling environment for researchers in public and private sector medical colleges/universities can help gather insight into the dynamics of policies and delivery of healthcare training to residents of one of the most populous countries of the world.

However, this study did have some limitations. A major limitation is that only the PubMed ${ }^{\circledR}$ (National Library of Medicine) database was searched as this might have excluded possible publications that met the inclusion criteria; this search was purposefully structured to include Pakistani journals not indexed in other databases. Focusing exclusively on mental health research in medical colleges/universities may have resulted in some studies being omitted if they had not been carried out by specialists not affiliated with a medical college/university. Future studies with broader inclusion criteria may provide more detailed results of the research achievements and impart a clearer idea of how to improve the research output in Pakistan

\section{Conclusion}

The current findings are encouraging as the quantitative as well as qualitative measures of mental health research in Pakistan have shown steady improvement over the last decade. However, certain policy changes are needed to cultivate a better research culture in Pakistan. The foremost change needed is to develop a system for funding research in medical education institutions.

\section{ACKNOWLEDGEMENT}

The authors would like to thank Dr Asma Humayun for her suggestions and feedback on the manuscript draft.

\section{CONFLICT OF INTEREST}

The authors declare no conflict of interest.

\section{FUNDING}

No funding was received for this study.

\section{References}

1. Mills A. Health care systems in low- and middle-income countries. N Engl J Med 2014; 370:552-7. https://doi.org/10.1 056/NEJMra1110897.

2. Green A, Rana M, Ross D, Thunhurst C. Health planning in Pakistan:Acasestudy.IntJHealth PlannManag 1997;12:187-205. https://doi.org/10.1002/(SICI)1099-1751(199707/09)12:3< 187::AID-HPM479>3.0.CO;2-0.

3. Institute of Medicine. The value, importance, and oversight of health research. In: Nass SJ, Levit LA, Gostin OL, Eds. Beyond the HIPAA Privacy Rule: Enhancing Privacy, Improving Health Through Research. Washington, DC, USA: National Academies Press, 2009.

4. Steinwachs DM, Hughes RG. Health Services Research: Scope and Significance. In: Hughes RG, Ed. Patient Safety and Quality: An Evidence-Based Handbook for Nurses. Maryland, USA: Agency for Healthcare Research and Quality, 2008. Pp. 1-15.

5. The Global Economy. Pakistan: Health spending as percent of GDP. From: www.theglobaleconomy.com/Pakistan/Health_sp ending_as_percent_of_GDP/ Accessed: Apr 2019.

6. Sumathipala A, Siribaddana S, Patel V. Under-representation of developing countries in the research literature: Ethical issues arising from a survey of five leading medical journals. BMC Med Ethics 2004; 5:e5. https://doi.org/10.1186/1472-6939-5-5.

7. Smith R. Publishing research from developing countries. Stat Med 2002; 21:2869-77. https://doi.org/10.1002/sim.1291.

8. Salager-Meyer F. Scientific publishing in developing countries: Challenges for the future. J English Acad Purp 2008; 7:121-32. https://doi.org/10.1016/j.jeap.2008.03.009.

9. Gaillard J. Use of publication lists to study scientific production and strategies of scientists in developing countries. Scientometrics 1992; 23:57-73. https://doi.org/10.1007/BF020 20914

10. Pittet D, Allegranzi B, Storr J, Bagheri Nejad S, Dziekan G, Leotsakos A, et al. Infection control as a major World Health Organization priority for developing countries. J Hosp Infect 2008; 68:285-92. https://doi.org/10.1016/j.jhin.2007.12.013.

11. Saraceno B, Saxena S. Mental health resources in the world: Results from Project Atlas of the WHO. World Psychiatry 2002;1:40-4.

12. Patel V, Sumathipala A. International representation in psychiatric literature: Survey of six leading journals. Br J Psychiatry 2001; 178:406-9. https://doi.org/10.1192/bjp.178.5.406.

13. The Patient Factor. World Health Organization's ranking of the world's health systems. From: www.thepatientfactor.com/cana dian-health-care-information/world-health-organizationsranking-of-the-worlds-health-systems/ Accessed: Apr 2019.

14. Ghaffar A, Zaidi S, Qureshi H, Hafeez A. Medical education and research in Pakistan. Lancet 2013; 381:2234-6. https://doi. org/10.1016/S0140-6736(13)60146-4.

15. Garfield E. The meaning of the impact factor. Int J Clin Heal Psychol 2003; 3:363-9.

16. Naqvi HA, Khan MM. Mapping exercise of mental health research and researchers in Pakistan. I Pak Med Assoc 2007; 57:294-8.

17. Pakistan Medical \& Dental Council. Regulations for the appointment/promotions of faculty professional staff/examiners/principals/administrative staff in undergraduate \& postgraduate medical \& dental institutions of Pakistan 2017. From: http://pmdc.org.pk/LinkClick.aspx?fileticket=tenGaMQG fbc\%3D\&tabid=404\&mid=1064 Accessed: Apr 2019. 
18. Junaidi I. Corruption allegations rock PMDC again. From: www.dawn.com/news/1325852 Accessed: Apr 2019.

19. King Edward Medical University. Home page. From: http:// kemu.edu.pk/ Accessed: Apr 2019.

20. College of Physicians \& Surgeons Pakistan. Home. From: www. cpsp.edu.pk/ Accessed: Apr 2019.

21. College of Physicians \& Surgeons Pakistan. Training programs. From: www.cpsp.edu.pk/fcps.php Accessed: Apr 2019.

22. Iqbal MP. What ails medical research in Pakistan? Role of institutions. Pak J Med Sci 2015; 31:1287-9. https://doi.org/10.1 2669/pjms.316.9337.
23. Qureshi MA, Qureshi MS, Khanani MR. Health and research in Pakistan. Lancet 2013; 382:1245. https://doi.org/10.1016/ S0140-6736(13)62091-7.

24. Nishtar S, Bhutta ZA, Jafar TH, Ghaffar A, Akhtar T, Bengali K, et al. Health reform in Pakistan: A call to action. Lancet 2013; 381:2291-7. https://doi.org/10.1016/S0140-6736(13)60813-2.

25. Burke JD Jr, Pincus HA, Pardes H. The clinician-researcher in psychiatry. Am J Psychiatry 1986; 143:968-75. https://doi. org/10.1176/ajp.143.8.968.

26. Kupfer DJ, Hyman SE, Schatzberg AF, Pincus H, Reynolds CF 3rd. Recruiting and retaining future generations of physician scientists in mental health. Arch Gen Psychiatry 2002; 59:657-60. https://doi.org/10.1001/archpsyc.59.7.657. 